\title{
Assessing the effect of interaction between an FTO variant (rs9939609) and physical activity on obesity in 15,925 Swedish and 2,511 Finnish adults
}

\author{
A. Jonsson • F. Renström • V. Lyssenko • E. C. Brito • \\ B. Isomaa • G. Berglund • P. M. Nilsson • L. Groop • \\ P. W. Franks
}

Received: 18 December 2008/Accepted: 16 March 2009/Published online: 17 April 2009

(C) Springer-Verlag 2009

\begin{abstract}
Aims/hypothesis Recent reports have suggested that genotypes at the FTO locus interact with physical activity to modify levels of obesity-related traits. We tested this hypothesis in two non-diabetic population-based cohorts, the first from southern Sweden and the second from the Botnia region of western Finland.

Methods In total 2,511 Finnish and 15,925 Swedish nondiabetic middle-aged adults were genotyped for the FTO
\end{abstract}

Electronic supplementary material The online version of this article (doi:10.1007/s00125-009-1355-2) contains supplementary material, which is available to authorized users.

\footnotetext{
A. Jonsson · V. Lyssenko · L. Groop

Department of Clinical Sciences-Diabetes and Endocrinology,

CRC, Malmö University Hospital MAS, Lund University,

Malmö, Sweden

F. Renström • E. C. Brito $・$ P. W. Franks $(\square)$

Genetic Epidemiology and Clinical Research Group,

Department of Public Health and Clinical Medicine,

Medicine Clinic, Umeå University Hospital,

Level 4, Stair B,

Umeå 901 87, Sweden

e-mail: paul.franks@medicin.umu.se

URL: www.umu.se/phmed/medicin/paul.franks/

B. Isomaa

Folkhälsan Genetic Institute,

Helsinki, Finland

B. Isomaa

Malmska Municipal Health Care Center and Hospital,

Jakobstad, Finland

G. Berglund - P. M. Nilsson

Department of Medicine, Malmö University Hospital MAS,

Lund University,

Malmö, Sweden

rs9939609 variant. Physical activity was assessed by questionnaires and standard clinical procedures were conducted, including measures of height and weight and glucose regulation. Tests of gene $\times$ physical activity interaction were performed using linear interaction effects to determine whether the effect of this variant on BMI is modified by physical activity.

Results The minor A allele at rs9939609 was associated with higher BMI in both cohorts, with the per allele difference in BMI being about 0.13 and $0.43 \mathrm{~kg} / \mathrm{m}^{2}$ in the Swedish and Finnish cohorts, respectively $(p<0.0001)$. The test of interaction between physical activity and the rs9939609 variant on BMI was not statistically significant after controlling for age and sex in either cohort (Sweden: $p=0.71$, Finland: $p=0.18$ ). Conclusions/interpretation The present report does not support the notion that physical activity modifies the effects of the FTO rs9939609 variant on obesity risk in the nondiabetic Swedish or Finnish adults studied here.

Keywords Gene $\times$ environment interaction $\cdot$ Gene $\times$ lifestyle interaction - Genetic $\cdot$ Physical activity $\cdot$ Obesity $\cdot$ FTO .

Swedish · Finnish

$\begin{array}{ll}\text { Abbreviations } & \\ \text { MET } & \text { Metabolic equivalent } \\ \text { MPP } & \text { Malmö Preventive Project } \\ \text { PPP-Botnia Study } & \begin{array}{l}\text { Prevalence, Prediction and Prevention } \\ \text { of Diabetes-Botnia Study }\end{array}\end{array}$

Introduction

The realisation that weight-gain is highly heritable [1] has prompted the search for and subsequent discovery of several genetic risk factors for common obesity $[2,3]$, the 
strongest of which localises to the fat mass and obesity associated gene (FTO) [4]. Each copy of the FTO risk allele, prevalent at $\sim 40 \%$ in whites, corresponds to $\sim 1.5 \mathrm{~kg}$ heavier weight [4]. Thus, the population attributable risk of obesity associated with the FTO variant is relatively high. An important question, therefore, is whether the deleterious effects of some FTO polymorphisms on obesity can be offset by lifestyle factors that might be open to intervention.

Three recent reports have suggested that physical activity [5,6] or lifestyle intervention [7] might offset the detrimental impact of FTO variants on obesity-related traits. In the present report we attempted to extend these findings by assessing the effect of interaction between the FTO rs9939609 variant and physical activity on BMI in 18,435 non-diabetic Swedish and Finnish adults.

\section{Methods}

The Malmö Preventive Project (MPP) was initiated in 1974. The aim was to screen the adult population of Malmö, a city in southern Sweden, in order to identify high-risk individuals for preventive interventions. Participants were invited to undergo a physical examination including anthropometry, a self-administered questionnaire on lifestyle habits and fasting blood sampling. From 1974 to 1992, 22,444 males and 10,902 females attended the screening programme (71\% participation rate). The majority of participants also underwent a follow-up examination, which occurred on average 23 years later (see Table 1). Both data points are included in the analyses. Analyses were conducted with $(n=15,844)$ and without $(n=13,799)$ the inclusion of incident diabetic individuals, although this made no material difference to the results.

The PPP (Prevalence, Prediction and Prevention of Diabetes)-Botnia Study is a population-based study in the Botnia region of western Finland that was initiated in 2004 in five research centres (Närpes, Malax-Korsnäs, Korsholm,
Vasa and Jakobstad). The study was designed to determine the prevalence and risk factors for type 2 diabetes within the community.

All participants gave written informed consent, and the studies were approved by the local research ethics committees of Lund University and Malmö University Hospital (MPP) and Helsinki University hospital (PPP-Botnia Study).

Clinical measurements The clinical examination included measurements of height $(\mathrm{m})$ and weight $(\mathrm{kg})$ as previously described using standardised methods $[8,9]$. BMI was calculated as weight divided by height squared $\left(\mathrm{kg} / \mathrm{m}^{2}\right)$. An OGTT was performed as previously described $[8,9]$. These measurements, in addition to clinical records, were used to ensure that all participants in the present report were nondiabetic at baseline.

Measurement of physical activity Physical activity was assessed using self-administered questionnaires. The MPP spanned a number of years, during which the physical activity questions changed. Therefore, we constructed a variable where an individual was classified as 'physically active' if they had answered 'yes' when responding to one of the following questions: (1) do you walk or cycle to and from work; (2) do you walk or cycle for recreation during weekdays; (3) do you walk or cycle for recreation during weekend days; (4) do you undertake at least $3 \mathrm{~h} /$ week of structured physical exercise; (5) do you walk to work or do yard work; or (6) do you perform light structured physical exercise each week. Conversely, those who answered 'no' to these questions were classified as 'physically inactive'.

In the PPP-Botnia Study, physical activity was assessed using the validated Kuopio Ischemic Heart Disease questionnaire [10]. This questionnaire estimates the duration, frequency, and mean intensity of leisure-time physical activity during the previous 12 month period. The questionnaire focuses on the structured and non-structured activities common to middle-aged Finnish men. In the

Table 1 Participant characteristics

\begin{tabular}{llll}
\hline Characteristic & MPP & & PPP-Botnia $(n=2,511)$ \\
\cline { 2 - 3 } & Baseline $(n=15,925)$ & Follow-up $(n=15,844)$ & \\
\hline Sex $(\mathrm{M} / \mathrm{F})$ & $10,330 / 5,595$ & $10,278 / 5,566$ & $1,185 / 1,326$ \\
Age $($ years $)$ & $45.5(6.9)$ & $68.5(5.6)$ & $46.5(15.6)$ \\
BMI $\left(\mathrm{kg} / \mathrm{m}^{2}\right)$ & $24.3(3.3)$ & $27.1(4.1)$ & $26.0(4.2)$ \\
Physically active & $3,451 / 12,480$ & $3,430 / 12,414$ & $573 / 1,304 / 634$ \\
rs9939609 (TT/TA/AA) & $5,557 / 7,656 / 2,718$ & $5,528 / 7,613 / 2,703$ & $957 / 1,169 / 385$ \\
\hline
\end{tabular}

Values are $n$ or means (SD). Physical activity in the MPP is coded as 'sedentary' or 'physically active'. Physical activity in the PPP-Botnia Study is coded as a 'low' ( $<10 \mathrm{MET}-\mathrm{h} /$ week), 'moderate' (10-40 MET-h/week) or 'high' ( $>40 \mathrm{MET}-\mathrm{h} /$ week) level of physical activity

$\mathrm{M}$, male; F, female 
present analyses, total physical activity is expressed as metabolic equivalent (MET)-hours per week (MET-h/week) grouped as sedentary ( $<10 \mathrm{MET}$-h/week), moderately active (10-40 MET-h/week) or very active ( $>40 \mathrm{MET}-\mathrm{h} /$ week). For the three activity levels, the following types of exercise are included: walking, yard work, fishing or hunting, and berry or mushroom gathering (low intensity); brisk walking, skiing, jogging, swimming, bicycling, ball games and forestry, etc (moderate to high intensity); and skiing, jogging, ball sports or forestry (vigorous intensity).

The correlation between physical activity and BMI $\left(\mathrm{kg} / \mathrm{m}^{2}\right)$ was statistically significant in the MPP $(r=-0.06 ; p<0.0001)$ and in the PPP-Botnia Study $(r=-0.04 ; p=0.04)$, indicating that both measures classified physical activity correctly and comparably. Although these correlations are low, the correlations between objectively assessed activity levels and these phenotypes is only moderately stronger [11], suggesting that the methods used here appropriately classify activity levels.

Genotyping The FTO rs9939609 genotyping was done using allelic discrimination with a TaqMan assay on the ABI7900 platform (Applied Biosystems, Foster City, CA, USA). The genotyping success rate was $>99 \%$, in HardyWeinberg equilibrium $(p>0.05)$ and with an error rate $<1 \%$ (determined from $>7 \%$ re-genotyping).

Statistical analysis Statistical analyses were conducted using SAS software (v9.1; SAS Institute, Cary, NC, USA). Hardy-Weinberg equilibrium was assessed using Haploview v4.0 (www.broad.mit.edu/mpg/haploview, accessed 1 November 2008). The Pearson correlation statistic was used to assess the relationship between physical activity and BMI. Generalised linear models were used to test gene $\times$ physical activity interactions for the rs9939609 variant, with BMI as the continuous dependent variable. Prospective models included baseline age, sex and BMI, an interaction term for baseline physical activity $\times$ genotype and duration of follow-up as independent variables, with follow-up BMI as the dependent variable. Logistic regression was used to test gene $\times$ physical activity interactions on a categorical obesity variable, defined according to the recommendations of the WHO [12]. All models were adjusted for age and sex and assumed an additive mode of genetic inheritance.

\section{Results}

Descriptive characteristics of the cohorts are reported in Table 1.

As reported elsewhere [13], the minor A allele at rs9939609 was associated with increased levels of BMI in the MPP and the PPP-Botnia Study $(p<0.00001)$. In the MPP cohort the minor A allele was associated with roughly a $0.13 \mathrm{~kg} / \mathrm{m}^{2}$ unit increase in BMI per allele [13]. In the PPP-Botnia Study, a stronger association between the A allele and BMI was observed (TT $=25.8 \mathrm{~kg} / \mathrm{m}^{2}, 95 \%$ CI $25.5-26.0 \mathrm{~kg} / \mathrm{m}^{2}$; TA= $26.0 \mathrm{~kg} / \mathrm{m}^{2}, 95 \%$ CI $25.8-26.3 \mathrm{~kg} / \mathrm{m}^{2} ; \mathrm{AA}=26.6 \mathrm{~kg} / \mathrm{m}^{2}, 95 \%$ CI $\left.26.2-27.0 \mathrm{~kg} / \mathrm{m}^{2} ; p<0.00001\right)$. The per allele increase in BMI in the PPP-Botnia Study was roughly $0.43 \mathrm{~kg} / \mathrm{m}^{2}$.

The test of interaction between physical activity and the rs9939609 variant on BMI was not statistically significant in the MPP $(p=0.71)$ or the PPP-Botnia Study $(p=0.18)$ (see Fig. 1a, b). Consistently, in models where obesity was defined using the WHO categorical definitions [12], there was no evidence of a statistically significant interaction in either cohort, nor was there evidence of an effect of interaction on change in BMI from baseline to follow-up in the MPP with $(p=0.27)$ or without $(p=0.49)$ the inclusion of incident cases of diabetes (Electronic supplementary material [ESM] Fig. 1).

The MPP is a somewhat leaner population than the Danish cohort featured in the original report [5]. Therefore, to test for interaction effects that might also be dependent on the level of obesity, we restricted our analyses to the 5,705 individuals with BMI $>25.0 \mathrm{~kg} / \mathrm{m}^{2}$, but this made no material difference to the cross-sectional baseline $(p=0.64)$ or prospective $(p=0.54)$ interaction effects.

\section{Discussion}

FTO harbours several strongly associated obesity-risk variants [4]. Two recent reports have suggested that physical activity modifies the effects of two of these variants (rs9939609 and rs1861868) on BMI levels [5, 6]. In a third report [7], a 1 year programme of intensive lifestyle intervention did not influence the effects of the FTO rs9939609 variant on weight change; however, the genetic effect on gain in subcutaneous adipose tissue during
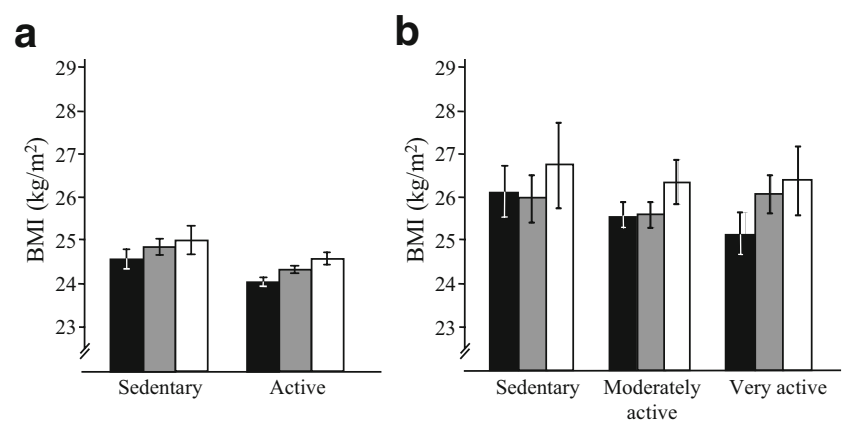

Fig. 1 Absence of an effect of interaction between an FTO singlenucleotide polymorphism (rs9939609) and physical activity on BMI in men and women from the baseline examination of the MPP $(n=$ 15,$\left.925 ; p_{\text {interaction }}=0.71\right)$ (a) or the PPP-Botnia Study $(n=2,511$; $\left.p_{\text {interaction }}=0.18\right)(\mathbf{b})$. Data are age- and sex-adjusted means $(95 \%$ CIs). Genotype groups: black bars, T/T; grey bars T/A; white bars A/A 
this period tended to be attenuated by intensive lifestyle intervention $(p=0.05)$. These reports are interesting as they suggest that it may be possible to offset the deleterious health effects of FTO variants through behavioural changes. Importantly, however, none of the published results can be considered as replicating the initial report, owing to different study designs [7] or loci examined [6]. Moreover, all three reports emanate from settings of multiple hypothesis testing. Thus, appropriately conducted replication attempts, such as those reported here, are important.

Here we examined whether physical activity modifies the effects of the FTO rs9939609 variant on BMI in a population-based study of 18,442 non-diabetic Swedish and Finnish adults. The aim of our study was to test the gene $\times$ physical activity interaction hypothesis proposed by Andreasen et al. [5]. Our study is about fourfold larger than the original report, meaning that with all else being equal it has sufficient power to detect the interaction effect reported previously [5]. Nonetheless, we found no evidence of an effect of interaction between the FTO variant and physical activity on levels of BMI.

As reported in other cohorts [4], the FTO variant was strongly associated with BMI in the present study and, as expected, physical activity was inversely related to BMI.

It is possible that our inability to replicate the initial study's findings is attributable to differences in the physical activity measures across studies or that the cohorts differ in other respects. The Danish and MPP cohorts are geographically proximal, comprising largely white, middle-aged northern Europeans, and similar physical activity metrics were used. However, the MPP participants were leaner on average than those in the Danish study and the effect of the FTO variant in the MPP is roughly half the effect reported elsewhere [3], possibly indicating that overall, MPP participants had relatively high habitual physical activity levels. This last point might explain the absence of interaction in the MPP. However, this is unlikely to explain the lack of a significant interaction effect in the PPP-Botnia Study, as this cohort is of comparable mean BMI to the Danish cohort. Moreover, restricting the MPP sample to people of similar BMI to the Danish cohort had no bearing on the interaction between rs9939609 and physical activity.

In conclusion, we were unable to replicate previous reports of gene× physical activity interactions at the FTO locus in large population-based cohorts of non-diabetic Scandinavian adults. The absence of an observable interaction in these cohorts does not necessarily rule out the possibility that previous observations of interaction are true, as there are several key distinctions between the cohorts studied here and those examined elsewhere [5]. One key distinction is that the Malmö cohort is relatively lean, which suggests absolute activity levels may be higher here than in other cohorts. This may impede the detection of the interaction effect. A relatively weak overall effect of the rs9939609 variant on BMI in this cohort supports this possibility. Ultimately an appropriate synthesis of available data including a range of activity levels and BMIs from a variety of cohorts should be feasible within the near future; this should allow one to reliably draw conclusions on whether physical activity attenuates the obesitypredisposing effects of the FTO rs9939609 variant. An important additional question that observational studies cannot address is whether lifestyle interventions modify genetic risk. Thus, a summary of evidence from relevant clinical trials, which to date include only two published studies [7, 14], should be undertaken when more become available. Finally, studies that examine whether other FTO variants interact with lifestyle behaviours may also be necessary to determine the extent to which the effects of FTO might be reduced or enhanced by specific behaviours.

Acknowledgements We thank those who participated in the present study as well as the Botnia Study Group. We are also grateful for the technical assistance of the staff of the CRC in Malmö. This work was funded in part by grants from the Swedish Diabetes Association, the Swedish Heart and Lung Foundation, Novo Nordisk, the Knut and Alice Wallenberg Foundation, the Påhlsson Foundation, the Sigrid Juselius Foundation, the Folkhälsan Research Foundation, the Nordic Center of Excellence in Disease Genetics, an EU grant (EXGENESIS), the Signe and Ane Gyllenberg Foundation, the Swedish Cultural Foundation in Finland, the Finnish Diabetes Research Foundation, the Foundation for Life and Health in Finland, the Finnish Medical Society, the Paavo Nurmi Foundation, Helsinki University Central Hospital Research Foundation, the Perklén Foundation, the Ollqvist Foundation and the Närpes Health Care Foundation. Some of the work reported in this paper was undertaken by P. W. Franks whilst a Visiting Scientist at the Harvard School of Public Health, Boston, MA, USA.

Duality of interest The authors declare that there is no duality of interest associated with this manuscript.

\section{References}

1. Bouchard C, Tremblay A, Despres JP et al (1990) The response to long-term overfeeding in identical twins. $\mathrm{N}$ Engl J Med 322:1477-1482

2. Willer C, Speliotes EK, Loos RJ, Li S et al (2009) Six new loci associated with body mass index highlight a neuronal influence on body weight regulation. Nat Genet 41:25-34

3. Thorleifsson G, Walters GB, Gudbjartsson DF et al (2009) Genome-wide association yields new sequence variants at seven loci that associate with measures of obesity. Nat Genet 41:18-24

4. Frayling TM, Timpson NJ, Weedon MN et al (2007) A common variant in the FTO gene is associated with body mass index and predisposes to childhood and adult obesity. Science 316:889-894

5. Andreasen CH, Stender-Petersen KL, Mogensen MS et al (2008) Low physical activity accentuates the effect of the FTO rs 9939609 polymorphism on body fat accumulation. Diabetes 57:95-101

6. Rampersaud E, Mitchell BD, Pollin TI et al (2008) Physical activity and the association of common FTO gene variants with body mass index and obesity. Arch Intern Med 168:1791-1797 
7. Franks PW, Jablonski KA, Delahanty LM et al (2008) Assessing genetreatment interactions at the FTO and INSIG2 loci on obesity-related traits in the Diabetes Prevention Program. Diabetologia 51:2214-2223

8. Berglund G, Nilsson P, Eriksson KF et al (2000) Long-term outcome of the Malmo preventive project: mortality and cardiovascular morbidity. J Intern Med 247:19-29

9. Lyssenko V, Jonsson A, Almgren P et al (2008) Clinical risk factors, DNA variants, and the development of type 2 diabetes. N Engl J Med 359:2220-2232

10. Lakka TA, Venalainen JM, Rauramaa R, Salonen R, Tuomilehto J, Salonen JT (1994) Relation of leisure-time physical activity and cardiorespiratory fitness to the risk of acute myocardial infarction. N Engl J Med 330:1549-1554
11. Franks PW, Ekelund U, Brage S, Wong MY, Wareham NJ (2004) Does the association of habitual physical activity with the metabolic syndrome differ by level of cardiorespiratory fitness? Diabetes Care 27:1187-1193

12. World Health Organization (2000) Obesity: preventing and managing the global epidemic. In: Technical report series 894 . World Health Organization, Geneva

13. Jonsson A, Franks PW (2009) Obesity, FTO gene variant and energy intake in children. N Engl J Med 360:1571-1572

14. Lappalainen TJ, Tolppanen AM, Kolehmainen M et al (2009) The common variant in the FTO gene did not modify the effect of lifestyle changes on body weight: the Finnish Diabetes Prevention Study. Obesity doi:10.1038/oby.2008.618 